\title{
Nuclei of Various Sizes and Shapes Present
}

National Cancer Institute

\section{Source}

National Cancer Institute. Nuclei of Various Sizes and Shapes Present. NCI Thesaurus. Code C83172.

A morphologic finding indicating the presence of a cellular population with a range of nuclear sizes and shapes. 